\title{
EREBEA
}

Revista de Humanidades

y Ciencias Sociales

Núm. 6 (2016), pp. 147-167

ISSN: 0214-0691

\section{La RECEPción de SHaKespeare EN LA COMUNidAd BRITÁNiCA en Minas de Riotinto y el Alentejo portugués}

\author{
María Dolores Carrasco Canelo \\ Universidad de Huelva
}

\section{RESUMEN}

Este trabajo propone revelar el desarrollo de dos bibliotecas victorianas, la "Biblioteca de Recreo" en el English Club of Rio Tinto en Minas de Riotinto (Huelva, España) y la biblioteca en el "Clube do Pessoal da Empresa Mason and Barry Ltd." en Mina de Santo Domingos (Mértola, Portugal). Estas se ubicaron en dos enclaves británicos mineros fuera de las fronteras de su Imperio, en la conocida Faja Pirítica Ibérica en el periodo en el que los británicos se establecieron en Minas de Santo Domingos (1858-1966) en el Alentejo portugués y Minas de Riotinto (18731954) en Huelva. Ambas albergan un relevante legado literario entre los que destaca la presencia del poeta y dramaturgo William Shakespeare y la identificación, vinculación e interacción con el auditorio lector que disfrutaba de sus ejemplares. La recopilación de datos y manuscritos sobre estas bibliotecas junto con estudios de recepción de este autor permiten entender la riqueza de estos grupos de volúmenes y el eje modélico que representaba el Bardo inglés cuyas obras se consideran extraordinarias como rastro de la huella británica en estos rincones del imperio.

Palabras Clave

Siglos XIX-XX; herencia británica en España y Portugal; literatura inglesa en enclave minero; biblioteca victoriana; Shakespeare.

Fecha de recepción: 25 de octubre de 2016 Fecha de aceptación: 30 de nov. de 2016

\section{ABSTRACT}

This paper seeks to reveal the development of two book collections from two Victorian libraries: the library at the English Club of Rio Tinto in Minas de Riotinto (Huelva, Spain) and the library at the "Clube do Pessoal da Empresa Mason and Barry" in Mina de Santo Domingos (Mertola, Portugal). They were located in two British mining sites that did not belong to the Empire, in the Iberian Pyrites Belt in the villages Mine of San Domingos (1858-1966) in southern Portugal and Minas de Riotinto (18731954) in Huelva. They prove with their catalogues a rich literary legacy of the British in the south of the Iberian Peninsula including the way in which Shakespeare's plays were portrayed and the interaction with the readers' wish to share the admiring image of the poet and playwright. The historical documents of these libraries, bearing in mind the critics' words when they regard the English writer, let us understand the relevance of these collections as well as the English Bard granted as an example of the British trace in this remote area of the Empire.

\section{KeYwords}

$19^{\text {th }}$ and $20^{\text {th }}$ centuries; British heritage in Spain and Portugal; English literature in mining sites; Victorian library; Shakespeare. 



\section{INTRODUCCIÓN}

"From Paramatta, from New York, wheresoever, under what sort of Parish-Constable soever, English men and women are, they will say to one another: 'Yes, this Shakespeare is ours; we produced him, we speak and think by him; we are of one blood and kind with him'”

Lecture III, The Hero as Poet, Dante Shakespeare

Thomas Carlyle ${ }^{1}$

Cuando la empresa británica Mason and Barry y el consorcio extranjero The Rio Tinto Company Limited se plantearon trasladarse a la Península Ibérica en pleno auge victoriano de la segunda mitad del siglo XIX para explotar los recursos mineros que estos parajes alejados de sus fronteras les ofrecían, consciente e inconscientemente, incorporaron en su equipaje todo su acervo cultural. Los pueblos donde se instalaron, Mina de Santo Domingos (1858) en Portugal en el caso de Mason \& Barry, y Minas de Riotinto (1876) en España, con The Riotinto Company Limited, experimentarían una metamorfosis radical como resultado del legado cultural, histórico y literario que la comunidad extranjera dejó tras de sí y que aún no ha sido debidamente atendido por parte del mundo académico. El presente trabajo pretende contribuir al estudio de dicha herencia con el análisis particular una de sus facetas menos conocidas.

En estas poblaciones, en esencia organizadas como colonias aunque formalmente nunca lo fueran, se instalaron comunidades de habla inglesa, en su mayoría británicas, que desde sus inicios se propusieron reproducir los hábitos y valores culturales propios de su sociedad victoriana, incluyendo la fundación de clubs sociales donde gestionarían la organización de bibliotecas para disfrutar de una amplia variedad de lecturas, desde la científica, específica y propia de sus labores empresariales y mineras, hasta la placentera y recreativa, que respondía al canon literario vigente en la época victoriana. Su estancia en estas tierras españolas y portuguesas ha dejado un rastro fiable que permite hoy indagar sobre la gestión

1Thomas Carlyle, On Heroes, Hero-worship and the Heroic in History. London: Chapman \& Hall, 1891, p. 105. 
y administración de las bibliotecas británicas y la recepción y difusión de obras y autores clásicos en la Faja Pirítica Ibérica en el siglo XIX.

Parte de las investigaciones que se llevan a cabo en torno a esa época en la actualidad se canalizan a través del denominado Neo-victorianismo, que permite una reinterpretación y reinvención de la era Victoriana por medio de nuevas vías de investigación. Ese es, pues, el marco teórico del que partimos para el estudio de la cultura victoriana en las comunidades de lengua inglesa establecidas en enclaves españoles y portugueses, un estudio que consideramos debe hacerse con nuevos ojos, tal como propuso Mark Lewellyn:

Neo-Victorian studies aims to tap into the potential for rereading, re-voicing, and re-imagining the collective memory of a global cultural moment. That this moment is still with us-in our municipal spaces, our collective identities, our parliamentary, educational, and social systems ... is self evident. ${ }^{2}$

Este marco crítico nos ofrece categorías e ideas que ayudan a entender y evaluar las implicaciones del modelo de lectura que se implantó en estos enclaves extranjeros. Lo victoriano se ha trasmitido canónicamente como una marca demasiado estereotipada, asociada a lo conservador, lo estricto y opuesto a cualquier propuesta renovadora y diferente. Si bien en muchos aspectos el estereotipo tuvo su validez, sí es importante señalar que parte del corpus estudiado en este artículo señala elecciones que complementaban a las puramente canónicas. Veremos, por ejemplo, cómo además de William Shakespeare, llegó a una de estas bibliotecas el volumen de The Girlhood of Shakespeare's Heroines, de Mary Cowden Clarke, que, a pesar de la popularidad que alcanzó en su época, recibió poca atención por parte de la crítica del momento. Altick señala al respecto: "The books she produced during those years are never recorded in the histories of Victorian literature, nor did they receive much mention in the contemporary press. Yet each enjoyed a notice-able degree of popularity in its day." ${ }^{3}$ La presencia de tales libros en lugares $\tan$ remotos parece indicar que estas bibliotecas de enclaves extranjeros podían incluso mostrarse más avanzadas en cuanto a la evolución del canon que el resto del país.

De ese modo, más allá de presentarnos el reverso cultural del proceso de explotación económica que está en el origen de las comunidades en que surgieron, las bibliotecas nos ofrecen una información muy valiosa sobre aspectos generales

2 Mark Lewellyn. "What is Neo-Victorian Studies?", en Neo-Victorian Studies, vol.1, n.1. Autumn (2008), 164-185, p. 180.

3 Richard D. Altick. The Cowden Clarkes. London, New York, Toronto: Oxford University Press, 1948, p. 143. 
de la cultura de la época (desde los libros que leían sus usuarios al método en el que tales libros se almacenaban), pero también del modo en que dicha cultura se veía preservada o alterada en las particulares circunstancias en que se encontraban sus miembros, ya que, por ser la lectura crucial para su bienestar físico y espiritual, los libros se convirtieron en medios de comunicación con la metrópoli y, por ello, revelaban a su modo la relación con los valores morales e ideológicos y también las modas que se sucedían en ella. Además, la documentación técnica de las bibliotecas nos ayuda a indagar en la formación técnica y moral de los individuos que las gestionaron y utilizaron porque, como mantienen Alistar Black y Peter Hoare, "as research subjects, marginal, small-scale libraries are as important as the large-scale collections".

\section{Primeros momentos: ANTECEDENTES E IMITACIÓN}

Canst thou remember

A time before you came into this cell?

I do not think thou canst.

\section{The Tempest, \\ William Shakespeare ${ }^{5}$}

La aventura comenzó a finales de la década de 1850 con la aparición de compañías extranjeras como Mason \& Barry (1858) en Mina de Santo Domingos y The Rio Tinto Company Limited (1876) en Minas de Riotinto. La transformación de estas localidades portuguesa y española en dos microcosmos británicos vino de la mano de una mentalidad colonial industrial. Los primeros ańos de estas poblaciones en la Península responden a un estilo de vida plenamente victoriano, que se contextualiza en esa economía productiva que historiadores como Sean Purchase han perfilado: "The early to mid-Victorian years, between roughly 1830 and 1875, were the triumphant years of the British self-confidence, and in these decades the Victorians established a buoyant but increasingly productive economy".

Estos nuevos inquilinos británicos se instalaron en barriadas diseñadas según sus referencias culturales y según las necesidades propias de la Inglaterra del siglo XIX. El barrio de "Bella Vista", en Minas de Riotinto, y la denominada "Zona

4 Alistair Black \& Peter Hoare. "Libraries and the Modern World", en Alistair Black et al., (eds.): The History of the Libraries in Britain and Ireland III. 1850 to 2000. Cambridge: Cambridge University Press, 2006, 7-20, p. 12.

5 William, Shakespeare. The Tempest. Oxford: Oxford University Press, 1994, 1.2. 38-40.

6 Sean Purchase. Key Concepts in Victorian Literature. Hampshire: Palgrave Macmillan, 2006, p. 5. 
dos Ingleses", que estratégicamente estaba situada en la zona administrativa de Mina de Santo Domingos, fueron pensados por y para los empleados extranjeros, y en ellas habitaba la comunidad británica, separada del resto de la población. En estas, la distribución del espacio interior refleja también las necesidades de socialización de los que la habitaban, habituales en las zonas inglesas de desarrollo industrial, desde pistas de tenis a templetes de música (en el caso portugués), y por supuesto, clubs sociales, un comportamiento que se ajusta al perfil "clubbable", con el que Alistair Black ha definido la sociedad inglesa del XIX”. Debido al ya citado mimetismo cultural y a las particulares necesidades de comunicación y socialización de sus miembros, estas "colonias británicas", como todavía se las conoce en la actualidad, incorporaron también bibliotecas como parte del English Club of Rio Tinto y del Palace del ingeniero principal de Mina de Santo Domingos.

Durante la segunda mitad del siglo XIX, la lectura se había convertido en una actividad muy presente en las diferentes clases sociales británicas y, especialmente en la clase media: "Between the 1850s and the 1890s educators and writers differentiated between reading that was passive or frivolous and reading that was serious, active, and conductive to self-development" ${ }^{\prime}$. Este interés por la lectura y las bibliotecas es un indicio de la creciente preocupación de la clase gobernante victoriana por las actividades de tiempo libre de la clase media y trabajadora, ya que se consideraba que, lejos de fomentar buenos hábitos, el ambiente de los pubs, bares y tabernas alimentaba la violencia, la prostitución y la bebida'. Entre las distintas actividades alternativas culturales y de entretenimiento sano que se propusieron como solución estaba la lectura a través de bibliotecas.

Desde 1824 distintas voces habían propuesto actuar en esta dirección, como Robert Stanley en su ensayo Essay on the Benefitial Direction of Rural Expenditure o James Silk Buckingham con su Public Institutions Bill de 1835. Ambos habían insistido en la necesidad de una oferta de infraestructuras como museos, salas de lectura y bibliotecas para la clase trabajadora como alternativa a los bares y pubs. Así, en los debates parlamentarios de 1850 se dijo que las bibliotecas eran "the cheapest police that could possibly be established" 10 . Un ejemplo significativo de esta campaña es la ilustración de William Benn incluida en 1886 en el volumen Free Public Libraries de Thomas Greenwood ${ }^{11}$. En dicha ilustración distinguimos

7 Alistair Black, "Libraries of Our Own”, en Black et al., (eds), The History..., p. 123.

8 Janet Badia,y Jennifer Phegley, (eds.). Reading Women. Literary Figures and Cultural Icons from the Victorian Age to the Present. Canada: University of Toronto Press, 2006, p. 133.

9 Friedrich Engels, The Condition of the Working Class in England in 1844. London: British Library, 1892, en: http://www.bl.uk/collection-items/the-condition-of-the-working-class-in-england-in-1844-by-friedrich-engels

10 Robert Snape, "Libraries for Leisure Time" en Black, Alistair et al., (eds.): The History of the Libraries in Britain and Ireland III. 1850 to 2000. Cambridge: CUP, 2006, 40-55, p. 40.

11 Thomas Greenwood, Free Public Libraries. Their Organisation, Uses, and Management. Lon- 
dos fachadas, una de una biblioteca pública, que ofrece educación y lectura, y otra de un pub, con imágenes típicas que representan el ocio, el alcohol y los juegos de mesa. Se presentan así dos conceptos rivales entre los que podía elegir el ciudadano, tal como se anunciaba en el título de la ilustración, The Rivals: Which shall it be? La visión de la cuestión implícita en la ilustración resulta claramente maniquea. En el caso de la biblioteca, los carteles indican "Library", "Reading Rooms", "Free Library and Institute" o "Lending library" y transmiten seriedad no solo en las emblemáticas esculturas del interior, como, por ejemplo, claro está, la imagen de Shakespeare, sino también en la urbanidad y distinción de los dos individuos que están en ella, uno en el interior y otro a punto de entrar, y especialmente en su disposición activa e interesada. Frente a ello, el pub es un edificio en el que encontramos un cartel anunciador clásico "The Red Lion", con anuncios sugerentes (Red Lion, Ale, etc.) y con letreros en las salas superiores que anuncian Bagatelles o Billiards. A su vez, el individuo que se retrata junto a este pub es un ciudadano en posición reclinada, ocioso, con actitud estática y fumando, que está acompañado por un perro sentado que lo observa igualmente quieto e inactivo. En general, se presenta claramente una disyuntiva dirigida a sugerir la mejor y más correcta manera de disfrutar del tiempo libre, en la que la biblioteca queda representada con connotaciones positivas como centro para desarrollar un hobby productivo y constructivo como la lectura y el intercambio de libros. Por todo ello, Snape considera que esta ilustración representa "a classic image of the Victorian concept of rational recreation in its promotion of reading as a leisure time activity" ${ }^{12}$.

Las bibliotecas en estos enclaves en el extranjero son una prueba más de la gran estima que se le tenía al conocimiento escrito como mecanismo de entretenimiento, es decir, una sociedad inmersa en el placer por la cultura escrita que considera la lectura y literatura como evidencias de prestigio, estatus y poder. Las bibliotecas fueron brotando por toda la geografía británica en contextos muy dispares. Como antecedentes más claros de las fundadas en las minas de la Penísula Ibérica podemos citar las de los centros mineros del sur de Gales: "The miners of South Wales have acquired a reputation as being "great readers" ${ }^{13}$. Según Baggs entre 1870 y 1939 se llegaron a fundar hasta 200 bibliotecas y salas de lectura de sociedades de mineros en el sur de Gales, de tal manera que cada asentamiento minero, por pequeño que fuera, contaba con una:

don: Simpkin, Marshall \& Co., 1886, p. 10.

12 Snape, "Libraries for Leisure Time", p. 40.

13 Chris M. Baggs, "Radical Reading? Working-class Libraries in the Nineteenth and Early Twentieth Centuries" Black, Alistair et al., (eds.): The History of the Libraries in Britain and Ireland III. Cambridge: CUP, 2006, 169-201, p. 174. 
(...) many South Wales coal-mining communities had looked to themselves to provide for the growing reading needs of their inhabitants. Libraries and reading rooms could help significantly in meeting these requirements, whether their impetus was educational, cultural or recreational. ${ }^{14}$

Como las surgidas en la Faja Pirítica Ibérica, su fundación surgió para dar respuesta a las necesidades de una comunidad generalmente ignorada por las instituciones gubernamentales. En el caso de las del sur de Gales se llegaron a constituir alrededor de un centenar de bibliotecas de esta naturaleza entre finales de la década de 1860 y comienzos de 1870 hasta 1914, fechas que coinciden con las del English Club of Minas de Riotinto y la del Palace de Mina de Santo Domingos. En el caso de las bibliotecas galesas su declive comienza con la Primera Guerra Mundial o, para la mayoría, con la gran depresión de finales de la década de 1920, que las obligó a ser "rescatadas" por bibliotecas públicas y otras entidades gubernamentales, mientras que otras, como las aquí estudiadas, consiguieron mantenerse en funcionamiento hasta bien entrada la segunda mitad del siglo XX.

Estas bibliotecas se identificaban con el continuo intento de auto-superación característico de la sociedad victoriana:

Self-help was an acknowledge and widely advocated element of Victorian social thinking and policy, but to this the South Wales mining valleys added various extra defining factors in their own. ${ }^{15}$

Su independencia y autonomía en la fundación y gestión marcó sus inicios. Sus condiciones geográficas eran peculiares, ya que se hallaban apartadas y aisladas unas de otras y alejadas de ciudades más grandes y desarrolladas, una circunstancia que las hacía independientes, al ser conscientes de lo limitado de sus infraestructuras y de su capacidad para el traslado de libros y que las igualaba con las de los asentamientos en el extranjero. En ese sentido, otro aspecto similar es el de la participación de los dueños y oficiales de las minas en su fundación y gestión por medio de su presencia en los comités organizadores junto con otras figuras encargadas de la formación cultural y espiritual: "Similarly, other influential members of the community, especially local ministers of religion and schoolteachers, were frequently drafted onto the book selection committee for predictable reasons" ${ }^{\prime 16}$.

14 Chris M. Baggs, "The Whole Tragedy of Leisure in Penury: The South Wales Miners' Institute Libraries during the Great Depression”, en Libraries and Culture 39 (2004), 115-36, p. 116.

15 Baggs , 2004, p. 116.

16 Baggs, 2004, p. 118. 


\section{LAS BIBLIOTECAS EN MINA DE SANTO DOMINGOS Y MINAS DE RIOTINTO}

Knowing I lov'd my books, he furnish'd me

From mine own library with volumes that

I prize above my dukedom

\section{The Tempest, William Shakespeare ${ }^{17}$}

En la actualidad se puede rastrear la huella de lo que fue el denominado "Clube do Pessoal da Empresa Mason and Barry Ltd.", "el club de personal de la empresa, creado por Mason and Barry para funcionarios de la empresa" ${ }^{18}$. Este club es otro ejemplo más de la presencia de seńas de identidad culturales británicas dentro del sistema de explotación minera establecido, en este caso por por Mason \& Barry, desde mitad del XIX, y de la política de la empresa de contribuir al progreso social y cultural por medio de la biblioteca. Esta biblioteca no cuenta con la variedad de documentación que se describirá en el caso de la del English Club of Rio Tinto por lo que no se puede aportar una línea temporal tan detallada sobre su gestión, administración y funcionamiento. Lo que sí está demostrado es que, como ocurrió también en Minas de Riotinto, en las instalaciones se diferenció claramente a la clase trabajadora nativa y a los trabajadores extranjeros de la empresa británica. La comunidad británica disponía de un edificio administrativo denominado "Palacio de Administración”, hoy convertido en el Hotel Minas de Santo Domingos, y en el que se han conservado, de forma deslavazada y fragmentaria, los restos de lo que constituyó la biblioteca, posiblemente personal del director de la mina en sus inicios y, posteriormente, del club. A ello hay que ańadir cuatro libros de registros, tres de los cuales se conservan en los archivos de la Asociación Serrâo Martins: dos libros de registros que incluyen una catalogación rudimentaria (solo figuran en ellos los títulos de los libros y los autores sin ningún dato de edición) y dos libros de registro de préstamos.

Con estos datos podemos esbozar de forma escueta que la vida de esta biblioteca se desarrolló en tres periodos diferentes. Un periodo inicial (1858-1892), del que data un libro de registro de préstamos que abarca hasta 1892, y los volúmenes conservados en la actualidad la sala denominada "Biblioteca Amélia" del Hotel Mina de Santo Domingos, que toma su nombre de la visita realizada por la esposa del rey Carlos I de Portugal (1863-1908) que ejerció su reinado de 1889 a 1908.

Si bien en el caso de los volúmenes de la biblioteca de Minas de Riotinto la mayor parte del legado se ha ordenado y está a cargo de la Biblioteca de la

17 Shakespeare, 1994, pp. 166-168.

18 Joâo Miguel Serrâo Martins. Patrimónios e Potencialidades da Mina de S. Domingos: O exemplo Erica andevalensis, Cabezudo y Rivera. Mértola: Fundaçao Serrâo Martins, 2013, p. 29. 
Universidad de Huelva, los libros conservados en el Hotel de Mina de Santo Domingos, están almacenados de manera arbitraria en cuatro estanterías que albergan y mezclan volúmenes antiguos con libros modernos. No existe catálogo u ordenamiento bibliotecario específico, por lo que no parece que la colección, anterior al catálogo del English Club of Rio Tinto, haya suscitado mucho interés.

A una segunda etapa, que podríamos denominar "periodo oscuro" (18931966) pertenece el libro de registro de préstamos conservado en el archivo de la Asociación Serrâo Martins, el cual recoge los movimientos de los libros de forma parcial, y solo desde 1928 a 1952. La tercera y última etapa correspondería a los años posteriores a 1966, fecha en que la compañía inglesa cesa su labor industrial en la zona y el rastro de la biblioteca se pierde hasta nuestros días.

A diferencia de la anterior, la biblioteca en Minas de Riotinto fue una parte bien definida del English Club of Rio Tinto y surgió inicialmente como una sala de lectura o reading room, tal y como documentan los estatutos que se conservan de la fundación del Club. En ese sentido, la biblioteca parece haber seguido la misma evolución que las de las comunidades mineras del sur de Gales, según recuerda Baggs: "The late 1860s were chosen as marking the establishment of the first reading rooms (rather than libraries) associated with the mining industry in South Wales. Many reading rooms grew into libraries ${ }^{19}$.

La evolución de esta entidad se puede analizar en tres etapas diferentes. Un periodo inicial, desde la fundación del Club en 1878 y la inauguración en 1903 del presente edificio del mismo y de su biblioteca, que deja de ser itinerante. Así se desprende de la crónica que dedica al acontecimiento el diario La Provincia: "Los estantes de la biblioteca encierran magníficas obras para todos los gustos (. . .) los mejores clásicos ingleses y de otros países" ${ }^{20}$.

La biblioteca de Riotinto era un elemento lógico como parte de un club inglés británico para el staff, tal y como ocurría en el Reino Unido de la época: "many working men's clubs possessed reading rooms and libraries, especially in the early part of the twentieth century" ${ }^{21}$. Si bien el término y concepto de "biblioteca" aparecen por primera vez en 1903 en La Provincia, la documentación más sustanciosa existente es la fechada a partir de 1917, cuando se produce la renovación de la biblioteca por parte de la Compañía, The Rio Tinto Company Limited, bajo la dirección de Walter Browning Spencer, uno de los presidentes más emblemáticos.

Esa fecha abriría una segunda etapa o periodo de la biblioteca, tal como queda atestiguado por los registros del Minute Book, libro de cartas y actas existentes

19 Chris M. Baggs, “The Miners' Libraries of South Wales from the 1860s to the 1939”, tesis sin publicar. Aberystwyth: University of Wales, 1995, p. 20.

20 "Baile en Rio-Tinto" en La Provincia, n. 7020, 6 Diciembre (1903), p. 2.

21 Baggs, 1995, p. 23. 
de la biblioteca del English Club of Rio Tinto. Tal libro se abre con una carta del director general, Walter Browing Spencer, del 5 de junio de 1917 informando al secretario del Club sobre cómo se debe llevar a cabo la administración y gestión de la que define como "nueva biblioteca"22 y comienza con el siguiente enunciado "NEW LIBRARY FOR THE CLUB LITERATURE, TECHNICAL BOOKS \& PAPERS" marcando la diversidad de lecturas que se ofrecería en las nuevas instalaciones, donde se recopilarían los volúmenes ya almacenados desde la fundación del Club y de su sala de lectura para la creación de esta "New Library". En tales documentos se definen tres campos diferentes: lectura, estudio y formación, en otras palabras, una lectura de recreo o literaria; otra técnica y específicamente orientada a la labor desempeñada en las minas y, finalmente, la lectura de los periódicos a los que el Club estaba suscrito.

Los documentos y actas que se han conservado muestran la buena organización con la que se llevó a cabo la gestión de la biblioteca. Tales documentos, que recogen información sobre la marcha diaria de la entidad, revelan, por ejemplo, que existía un comité independiente de los demás organismos del Club, con miembros como Caddick, Hall y Curry, que era responsable del sistema de catalogación y distribución de los ejemplares. Más relevante aún son los datos recogidos sobre la variedad de usuarios de esta biblioteca, que estaba reservada a los socios del Club, los trabajadores de la Compañía, y a sus familias: "As it is intended that the members of the Staff and their families should have the greatest facilities for obtaining books" ${ }^{23}$. De ahí que existan en el catálogo diferentes géneros, volúmenes de aprendizaje, de lectura infantil, novelas juveniles y volúmenes para formación propios del público femenino, es decir, atalayas intelectuales muy variopintas, como también lo eran los volúmenes que se recibían de y sobre un mismo autor, como, según veremos más adelante, el que nos ocupa en este artículo, William Shakespeare.

La tercera y última etapa de esta biblioteca corresponde al periodo español que se abre a partir de 1954 y en el que se aprecia una clara imitación de los modelos ingleses en los primeros años de gestión española: "No habían cambiado las normas; se había cambiado de dueño" 24 . La gestión de la biblioteca en la etapa española se caracterizó, como era lógico, por la compra de una mayor cantidad de libros en español. Las primeras comisiones de gobierno del Club bajo tutela española se reunieron en el otoño de 1954 y algunos documentos con fecha del 25 noviembre de 1954 dan fe del cambio de orientación, recogiendo contactos con editoriales españolas para la compra de libros en español. A partir de esa

22 Minute Book. Riotinto: Biblioteca del English Club, s.d., p.2.

23 Minute Book, p.2

24 Eloy Navarro et al., (eds). "A Real Civilization" El legado británico en la provincia de Huelva. Huelva: Ayuntamiento de Punta Umbría, 2009, p. 302. 
fecha disminuye considerablemente la adquisición de libros en inglés, unos libros ahora, en comparación con los de la etapa inglesa, muy diferentes en naturaleza.

Estas dos bibliotecas muestran el esfuerzo de la comunidad trasladada a estos enclaves no sólo por mantener una cierta actividad intelectual, sino también por mantenerse en contacto con la cultura de la metrópoli, todo ello como resultado de una especie de auto-obligación moral y educativa, y en contraste con la labor meramente económica y material que las había llevado hasta allí, contradiciendo así en cierto modo la imagen trazada por el parlamentario Robert Lowe de New South Wales cuando describía al colonizador como "He does not buy books, pictures (...) the money-making colonist".25.

\section{Los CATÁLOgos de las Bibliotecas}

O let my books be then the eloquence s And dumb presagers of my speaking breast.

\section{Sonnet 23, William Shakespeare ${ }^{26}$}

El actual catálogo de la biblioteca de Mina de Santo Domingos cuenta con 105 libros conservados en la "Biblioteca Amélia" y cuatro volúmenes que se custodian en el Archivo de la Fundación Serrâo Martins. Los dos libros de catalogación existentes, que también se conservan en esta fundación, ponen de manifiesto que esta biblioteca no tenía parangón en la zona en la época que se gestionó, ya que llegó a contar con casi 3.000 ejemplares enumerados, a pesar de no estar completo el listado, una cifra mucho más elevada que la de los libros conservados.

El volumen total del catálogo de la biblioteca de Minas de Riotinto que debió ser mucho mayor de lo que es en la actualidad, por causa a las numerosas pérdidas que debieron producirse a lo largo del tiempo. Y no sólo porque la biblioteca se gestó, como se ha visto, en varias etapas y en diferentes edificios, sino porque los documentos consultados revelan diferentes destinos, ventas, donaciones o préstamos no devueltos (como los que se enviaron a la biblioteca del Huelva Club en 1925 (Carta del secretario del comité de biblioteca al secretario del Club Colón, del 22 de octubre de 1925, Minute Book, 19), así como la segregación en distintas ubicaciones, muchas de ellas aún por determinar, que hacen del catálogo real un ente todavía desconocido en su totalidad. Es también un catálogo fragmentado, ya que en ocasiones solo se cuenta con uno o dos volúmenes de

25 Robert Lowe, "On the Death of Richard Windeyer (Extract from a parliamentary address)", en Leonie Kramer and Adrian Mitchell (eds.), Oxford Anthology of Australian Literature. Melbourne: Oxford University Press, 1985, p. 26.

26 William Shakespeare.Shakespeare’s Sonnets. Madrid: Visor Libros D.L., 2011. 
toda una colección como las de Washington Irving y Eurípides. En la actualidad los libros se hallan repartidos en tres ubicaciones: la mayor parte, un conjunto de 2.052, llegaron a la biblioteca de la Universidad de Huelva en régimen de donación en 1999, pudiéndose consultar en la actualidad, en régimen de préstamo reducido, solo 1.771 de ellos, puesto que el resto fue eliminado por estar en mal estado. A este fondo hay que añadir los 253 libros de la actual biblioteca del Club Inglés Bella Vista, así como otros 172 de la Casa número 21 de Bella Vista, una casa victoriana que se muestra en la actualidad a los visitantes como ejemplo del legado inglés en Riotinto.

Bajo el mandato británico, en ambas bibliotecas se solicitaban principalmente libros en inglés, con algunos volúmenes en francés. En el caso de la de Minas de Riontinto, los libros se clasificaron atendiendo a distintas categorías que se muestran en los cajones del fichero conservado en el actual edificio del Club: 1) Biography, History, 2) Fiction, 3) Literature, Poetry, Theology, y 4) Art, Sports, Travels. A pesar de no contar con datos similares para la biblioteca de Mina de Santo Domingos, los volúmenes conservados ponen de manifiesto que cubrían los mismos géneros e intereses. Estas categorías coinciden en su mayoría curiosamente con las de los 20 catálogos de bibliotecas de clubs e institutes de mineros que estuvieron activos entre 1860 y 1939 y que han sido estudiados por Baggs. En ellos, la categoría Travel incluía títulos de geografía, mientras que la categoría de Fiction era la más numerosa, como documenta Braggs de manera evidente en el caso de la biblioteca de Tredegar, localidad del sureste de Gales, en la que el siguiente apartado más numeroso era el de Biography, History ${ }^{27}$. S.V.R. Lee, por su parte, que analiza las bibliotecas de los workingmen's clubs, prueba que eran abastecidas por una circulating library central de la Union que comenzó a funcionar en 1865 y en 1869 , y cuyo catálogo se organizaba atendiendo a las siguientes categorías, que siguen muy de cerca las de Riotinto y Santo Domingos:

It was divided into the following subject sections: a) Biography and History, Memoirs etc, b) Topography, travels, and voyages, c) Moral philosophy and religion, d) Political and social e) Science, natural history, and art, f) fiction, g) Poetry and drama h) Miscellaneous. $^{28}$

En este contexto, una pieza central del puzle eran las entidades que suministraban los ejemplares. The Times Book Club, junto con la Boot's Booklovers's Library, Mudie's Select Library y Smith fueron las principales circulating libraries

27 Baggs, 1995, p. 341.

28 S.V. R. Lee, "The Development of Libraries within the Working Men's Club and Institute Union”, tesis sin publicar. Aberystwyth: University of Wales, 1992, p. 85. 
que suministraron libros a las bibliotecas de las colonias o lugares apartados de las fronteras nacionales. De estas, tan solo las dos primeras, que fueron las que se mantuvieron activas a lo largo del siglo XX, aparecen en las actas de la biblioteca del English Club of Rio Tinto. Mudie's cerró en 1937, Smith en 1961, The Times Book Club en 1962 y Boot's Booklovers's Library en 1966, hecho que afectó a toda la red de bibliotecas que dependía de su suministro. Sin embargo, la biblioteca del English Club of Rio Tinto no se vio perjudicada pues había pasado a manos españolas en 1954 y había dado por terminadas sus relaciones comerciales con The Times Book Club y Boot's Booklover's Library antes de que estas desaparecieran. En Mina de Santo Domingos la principal suministradora fue Mudiés Select Library, que había sido fundada en 1842 y que en 1890, cuando falleció su fundador, contaba con 25.000 suscriptores, entre los que se encontraba esta biblioteca, aunque desconocemos hasta qué fecha se mantuvo el intercambio de ejemplares. Los encargados de estas bibliotecas intentaron reunir un canon literario tradicional que representara aspectos de la cultura inglesa al más puro estilo victoriano incluyendo figuras canónicas como William Shakesperare y que a continuación estudiamos.

\section{WILLIAM SHAKESPEARE, ICONO DEL IMPERIO}

"He is the grandest thing we have yet done. For our honor among foreign nations, as an ornament to our English Household, what item is there that we would not surrender rather than him? Consider now, if they asked us, Will you give up your Indian Empire or your Shakespeare, you English; never have had any Indian Empire, or never have had any Shakespeare? Really it were a grave question. Official persons would answer doubtless in official language; but we, for our part too, should not we be forced to answer: Indian Empire, or no Indian Empire; we cannot do without Shakespeare! Indian Empire will go, at any rate, some day; but this Shakespeare does not go, he lasts forever with us; we cannot give up our Shakespeare!"

Lecture III, The Hero as Poet, Dante Shakespeare

Thomas Carlyle ${ }^{29}$

El desarrollo de estas bibliotecas es una consecuencia del imperialismo británico, y se puede explicar si atendemos a una serie de oposiciones binarias de conceptos opuestos sobre la que se asienta la conciencia victoriana: lo propio y lo 
extraño, civilización y barbarie, colonizador y colonizado. Basándonos en estos conceptos, podemos ver cómo la literatura inglesa desempeńó un rol activo a la hora de levantar muros culturales y morales tras de los que el británico del siglo XIX se sentía más seguro y cómodo en toda la extensión del Imperio por todo el globo frente a los pueblos con los que se veía obligado a convivir. David Thelen ha definido así el proceso: "how cultures establish traditions and myths from the past to guide the conduct of their members in the present" ${ }^{\text {". }}$. La sociedad británica en el extranjero podía contrastar escenas, nociones, hábitos, pero, sobre todo, necesitaba reafirmar su cultura e identidad. Tengamos en cuenta que cuando el colonizador se establece en la periferia de otro país colonizado sufre, en gran medida, un proceso de otredad y alienación. El colonizador británico victoriano se diferencia del colonizado al considerarlo "el otro", pero irónicamente, el mismo colonizador sufre también un cierto distanciamiento en relación a su propia cultura e identidad al hallarse dentro de las fronteras del país ocupado. Y es ese empeño del colonizador por mantener los vínculos con su cultura y evitar convertirse en un "outsider" dentro de ella, que le hace aferrarse a sus señas de identidad y explica cómo y por qué las piezas de William Shakesperare encontraron su hueco en las bibliotecas británicas de las colonias en pleno auge del Imperio. Tal como ha señalado Purchase ${ }^{31}$ fue sobre todo en los países colonizados en los que Shakespeare, en tanto emblema de la cultura inglesa, se convirtió en lo que Gauri Viswanathan ha llamado "mask of conquest" 32 .

Los casos aquí estudiados presentan una marcada peculiaridad. En estas bibliotecas no podemos hablar de colonialismo o imperialismo cultural, puesto que las comunidades británicas en el sur de la Península Ibérica no impusieron su cultura a la sociedad autóctona. Crearon su propio hogar con marcas definidas de su identidad nacional en torno a obras clásicas, como las de su dramaturgo más reconocido, al que perciben la representación más explícita de su cultura, su idioma y su identidad, especialmente desde finales del siglo XVIII y a lo largo del siglo XIX. Hunt habla de la "canonisation of Shakespeare as the pre-eminent national dramatist"33, y Marshall describe la recepción del autor en el siglo XIX en el contexto en el que se establece la National Portrait Gallery en 1856, con la

30 David Thelen.. 'Memory and American History', en The Journal of American History, v. 75, n. 4, March (1989), 1117-1129, p. 1117.

31 Purchase, p. 228.

32 Gauri Viswanathan, Masks of Conquest: Literary Study and British Rule in India. New York: Columbia University Press, 1989.

33 Arnold Hunt. "Private Libraries in the Age of Bibliomania"en Glies Mandelbrote et al. (eds.): The History of the Libraries in Britain and Ireland II: 1640 to 1850. Cambridge: Cambridge University Press, 2006, 459-478, p. 448. 
primera adquisición del retrato Chandos y la apertura del Shakespeare Memorial Theatre en Stratford en $1879^{34}$.

Estas comunidades británicas leían, como se ha dicho, materiales relacionados con sus actividades profesionales en volúmenes sellados con ex libris que marcaban claramente el departamento al que pertenecían. Pero además de esta lectura técnica, los documentos de ambas bibliotecas suministran una rica información sobre el acceso que tenían a las lecturas recreativas. En ese terreno, Shakespeare es sin duda, junto a Scott y Dickens, el autor favorito en ambas comunidades, que muestran así ser parte de ese proceso que Stanley Wells ha definido como la "absorption of Shakespeare into the bloodstream of European civilization" 35 .

Desconocemos si estas bibliotecas contaron con algún generoso donante benefactor como Sir George Grey, "who was an ardent bibliophile"36 y que, tras siete ańos como Gobernador de la Cape Colony (1854-1861) y antes de trasladarse a Nueva Zelanda para desempeñar el mismo puesto político, donó en 1861 a la South Africa Library su colección de 3.500 volúmenes, entre los que se encontraban "the first and second folios of Shakespeare" 37 .

En los centros que aquí se estudian la presencia de Shakespeare fue más modesta. En la biblioteca de Mina de Santo Domingos, no obstante,se conservan volúmenes de la colección de Johnson y Steven de $1793^{38}$.

34 Gail Marshall (ed.). Shakespeare in the Nineteenth Century. Cambridge and New York, Cambridge University Press, 2012.

35 Stanley Wells, "Forward" en A. Luis Pujante, A \& Hoenselaars, Ton (eds.). Four Hundred Years of Shakespeare in Europe. Newark: University of Delaware Press, 2003, p. 9.

36 R. F. M. Immelman, "Libraries Abroad: "The 150 $0^{\text {th }}$ Anniversary (1818-1968) of the South African Library, Cape Town”, en Miles Journal of Library History, vol. Jan 1, 5, 1 (1970), 73-79, p. 2.

37 Immelman, p. 2.

38 Johnson's and Steven's Shakespeare. Prolegomena. In fifteen volumes, vol. I. London: Longman, 1793; Johnson's and Steven's Shakespeare. Prolegomena. In fifteen volumes, vol. II. London: Longman, 1793; Johnson's and Steven's Shakespeare. Glossarial Index, Tempest, Two Gentlemen of Verona, Merry Wives of Windsor. In fifteen volumes, vol. III. London: Longman, 1793; Johnson's and Steven's Shakespeare. Twelfth Night, Measure for Measure, Much Ado about Nothing. In fifteen volumes, vol. IV. London: Longman, 1793; Johnson's and Steven's Shakespeare. Winter's Tale, Comedy of Errors, Macbeth. In fifteen volumes, vol. VII. London: Longman, 1793; Johnson's and Steven's Shakespeare. King John, King Richard II, King Henry IV, Part I. In fifteen volumes, vol. VIII. London: Longman, 1793; Johnson's and Steven's Shakespeare. King Henry IV, Part II, King Henry V, King Henry VI, Part I. In fifteen volumes, vol. IX. London: Longman, 1793; Johnson's and Steven's Shakespeare. King Henry VI, Part II, King Henry VI, Part III, Dissertations, King Richard III. In fifteen volumes, vol. X. London: Longman, 1793; Johnson's and Steven's Shakespeare. King Henry VIII, Troilus and Cressida, Timon of Athens. In fifteen volumes, vol. XI. London: Longman, 1793; Johnson's and Steven's Shakespeare. Coriolanus, Julius Caesar, Antony and Cleopatra. In fifteen volumes, vol. XII. London: Longman, 1793; Johnson's and Steven's Shakespeare. Cymbeline, Titus Andronicus, Pericles. In fifteen volumes, vol. XIII. London: Longman, 1793; Johnson's and Steven's Shakespeare. King Lear, Romeo and Juliet. In fifteen volumes, vol. XIV London: Longman, 1793. 
Los restos de la biblioteca de Minas de Riotinto no ofrecen una colección tan prestigiosa, sino simples ejemplares de bolsillo al uso dirigidos a un público mucho más variado. En la biblioteca de la Universidad de Huelva están a disposición del lector seis volúmenes: Macbeth (London: Sands \& Co., [s. d.]), dos ejemplares de Shakespeare's Comedies (London: J. M. Dent and Co.; New York: E. P. Dutton \& Co., 1906), Shakespeare's Historical Plays, Poems \& Sonnets (London: J. M. Dent and Co.: New York: E. P. Dutton \& Co., [s. d.]), Shakespeare's Julius Caesar, Edited by E. F. Horsley B. A. (London: Toronto: J. M. Dent \& Sons., [s. d.]), y Shakespeare's Tragedies (London: J. M. Dent and Co.: New York: E. P. Dutton \& Co.: New York: E. P. Dutton \& Co. ([1906?]). El interés por Shakespeare se mide igualmente en el número de ejemplares de algunas de las citadas ediciones, ya que en estas bibliotecas se intentaban evitar las repeticiones, como indica una carta de 1925 de petición de libros a Londres: "As we already have a considerable number of books, it would naturally suit us better if we could have a voice in the selection of fresh ones to avoid any possible duplication"39.

La relevancia otorgada a Shakespeare se pone asimismo de relieve en la existencia de volúmenes críticos sobre su vida y sus obras, en concreto, dos ejemplares de la mencionada obra de Mary Cowden Clarke (1809-1898) The Girlhood of Shakespeare's Heroines, (London: J. M. Dent and Co.: New York: E. P. Dutton \& Co., [1906]); otros dos ejemplares de de William Hazlitt (17781830) Characters of Shakespeare's Plays (London: J. M. Dent and Co.: New York: E. P. Dutton \& Co., 1906) y finalmente un ejemplar de Charles Lamb (1775-1834) Tales from Shakespeare (London: J. M. Dent and Co., 1906). Mark Hollingsworth ${ }^{40}$ cubre el amplio campo de los estudios sobre Shakespeare del periodo victoriano, incluyendo sociedades sobre el Bardo inglés y el desarrollo de estudios académicos y recreativos sobre este autor con figuras como A. C. Bradley, Mary Cowden Clarke, F. J. Furnivall, Anna Jameson, Charles and Mary Lamb, Algernon Swinburne y Oscar Wilde. Estos libros sobre el dramaturgo atendían a diferentes perfiles sociales e intelectuales que se encontraban presentes en la comunidad extranjera, hecho que pone de manifiesto cómo esta sociedad valoraba la literatura y consideraba que tanto adultos como jóvenes debían tener todos las mismas posibilidades de acceso a las lecturas, y, de un modo u otro, al universo literario de Shakespeare.

The Girlhood of Shakespeare's Heroines llegó a ser un libro muy popular en el siglo XIX. Altick contabiliza 21 ediciones entre 1852 y 1906 y mantiene que alcanzó "great success on both sides of the Atlantic" ${ }^{\text {11 }}$. Estos cuentos, que solían trasmitirse

39 Minute Book, p. 20.

40 Mark Hollingsworth, "Shakespeare Criticism”, en Marshall (ed.), 2012, pp. 39-59.

41 Altick, p. 138. 
de generación en generación, se habían concebido para el disfrute y diversión de los lectores en un estilo puramente victoriano según las palabras de Caramanna:

In The Girlhood, Cowden Clarke explores Shakespeare's heroines in a style that was designed for Victorian readers. Her writing reflects the values of her culture. She uses flowery, ornate language to describe characters, occurrences, and scenes throughout The Girlhood. The distinct narrative voices uses enhancing adjectives and descriptive phrases to detail the heroines and surrounding characters. Her descriptions contain metaphors and hyperboles in an attempt to create sentimental expressions of character and circumstance. ${ }^{42}$

A esto hay que añadir que, según el artículo de Claudia Nelson ${ }^{43}$, "Growing Up: Childhood", los victorianos prestaron una especial atención a la infancia, de tal forma que se pretendía ofrecer herramientas para garantizar la felicidad de niños y niñas en esta faceta de la vida en lugar de precipitarlos a la edad adulta. Sobre esta misma cuestión, Caramanna afirma:

This stage gained importance, and literature written for children began to appear in Victorian homes. Increasing literacy rates, printing technologies, and provisions for transportation and production made children's books more accessible than they had been in the past (...) As the "Victorians inherited a growing concern about children" and as childhood increasingly became a more significant period of life, the child's literature book became much more of a social norm, and so did female authors. ${ }^{44}$

Cowden Clarke se inspiró para escribir The Girlhood en su relación y conversaciones con otros eruditos y amantes de Shakespeare, en especial, con Mary Lamb, que escribió junto a su hermano Charles Tales From Shakespeare publicado en 1807. Aunque Mary Lamb era mayor que Cowden Clarke ambas autoras intimaron y la última llegó a leer Tales From Shakespeare siendo apenas una jovencita. Con su personal homenaje a Shakespeare, además de estudiar a sus heroínas, potenciaba, como muchos autores victorianos, al autor como

42 Jillian Caramanna, "The Backwards Making of A Heroine: Mary Cowden Clarke's Girlhood and Its Importance in the Shakespearean Conversation", English Honors Thesis. University at Albany. State University of New York, 2011, p. 6.

43 Claudia Nelson, “Growing Up: Childhood”, en Herbert f. Tucker, Malden (eds.): A Companion to Victorian Literature and Culture. Oxford: Blackwell Publishing, 1999, 69-81.

44 Caramanna, pp. 10-11. 
representación del espíritu de la nación y transmisor de valores morales adecuados para las siguientes generaciones:

The Victorians believed that Shakespeare's plays contained important moral lessons. The Girlhood was a didactic piece of Victorian literature. It was enjoyable reading for young girls; however, it served a larger purpose. As the heroines developed, the circumstances of their childhoods, along with their actions and responses to these circumstances, demonstrated the behaviors and moral lessons that the Victorians saw as so important in the plays. ${ }^{45}$

Otro volumen en la biblioteca de Riotinto es la obra de Hazlitt publicada en 1817, Characters of Shakespeare's Plays, uno de los primeros estudios del siglo XIX en lengua inglesa sobre los dramas de Shakespeare, el cual, junto con otras obras, como las de Coleridge (también presente en este catálogo), contribuyó a impulsar la creciente estima pública por la genialidad del dramaturgo, al estar concebido como una guía instructiva para un lector adulto y cubrir todas las obras de Shakespeare. Como indica Patterson, la caracterización de los personajes y sus sutilezas psicológicas "in the light of the Romantic fascination with the individual and with the world within" ${ }^{\prime 4}$ eran los aspectos que más habían despertado la atención de Hazlitt como crítico.

Complementa la colección de Shakespeare el clásico Tales from Shakespeare, de los hermanos Lamb, Mary y Charles, serie que rescribe las obras de Shakespeare transformándolas en textos más apropiados y comprensibles para jóvenes lectores, modificando entre otros aspectos los actos actos violentos, muertes y otros elementos confusos o poco apropiados para el público al que iba destinada la obra. Mary Lamb fue la responsable de redactar la parte referida a las comedias y Charles la correspondiente a las tragedias tal y como afirma Caramanna: "Mary Lamb took the direct texts of Shakespeare's plays and transformed the language as to make them attractive, accessible, and understandable for children" ${ }^{47}$. En su afán por extender el público de Shakespeare, ambos hermanos plantearían asimismo la posibilidad de hacer a Shakespeare accesible también a las mujeres,

45 Caramanna, p. 17.

46 Charles Patterson, "Hazlitt's Criticism in Retrospect", en I Studies in English Literature, 1500-1900, vol. 21, n. 4, Fall (1981), 647-663, p. 648.

47 Caramanna, p. 8-9. 
un aspecto importante que ha sido estudiado con detalle por algunos críticos, como Poole ${ }^{48}$, Novy ${ }^{49}$ o Ciraulo ${ }^{50}$.

No es extraño tampoco encontrar en esta biblioteca una edición antigua de la obra de Carlyle, On Heroes, Hero-worship and the Heroic in History (London: Chapman \& Hall, 1891). En la exaltación de la figura de William Shakespeare que lleva a cabo en la obra, el autor confiere al dramaturgo un particular simbolismo imperial, como vínculo de unión para todos los ciudadanos británicos dispersos por el ancho Imperio: "there will be a Saxondom covering great spaces of the Globe. And now, what is it that can keep all these together into virtually one Nation? (...) This king Shakespeare" ${ }^{\text {"1 }}$. Shakespeare se revela en este libro como nexo cultural, símbolo de dignidad y civismo para estas comunidades coloniales en las que se repartían los ciudadanos victorianos británicos en el XIX. Por otra parte, la importante presencia del volumen de Coleridge, Coleridgés Essays \& Lectures on Shakespeare \& Some Other Old Poets \& Dramatists (London: J. M. Dent and Co.: New York: E. P. Dutton \& Co., [1907]) revela el interés de algunos lectores de la biblioteca de Riotinto por la crítica y el análisis literario.

Por lo tanto, la presencia de volúmenes de y sobre Shakespeare en estas bibliotecas de enclaves mineros como Minas de Riotinto y Mina de Santo Domingos son una muestra de la importancia que tenía la figura de Shakespeare en los hábitos de lectura de los británicos en el extranjero en el siglo XIX, ya se tratase de los ingenieros y parte del staff de las empresas británicas o de sus círculos familiares. Entre este último grupo, el público femenino utilizó con asiduidad estas bibliotecas. Como ha mostrado parte de la crítica reciente, la mujer en el XIX era ya una fiel lectora de Shakespeare. Flint, por ejemplo, ofrece en el capítulo "Reading and the family" 52 datos de la lectura de Shakespeare por parte de jóvenes guiadas por sus padres. Tal fue el caso de Dora Montefiore (18511933), sufragista y socialista anglo-australiana, que aprendió de los hábitos de su progenitor: "She recalled her father's reliance on Shakespeare and his practice of looking up Shakespeare's views on any topic which came up with conversation"53, o el de la importancia de la educación lectora sobre Shakespeare y Dumas que la escritora inglesa Eleanor Farjeon (1881-1965) recibió de su padre. ${ }^{54}$

48 Adrian Poole. Lamb, Hazlitt Keats. Great Shakespeareans. Volumen IV. London: Bloomsbury Arden Shakespeare, 2013.

49 Marianne Novy. Women's Re-visions of Shakespeare: On the Responses of Dickinson, Woolf, Rich, H.D., George Eliot and Others. Chicago: University of Illinois Press, 1990.

50 Darlene Ciraulo, "Fairy Magis and the Female Imagination: Mary Lamb's "A Midsummer Night's Dream” en Philological Quarterly, vol. 78, n. 4 Fall (1999), pp. 439-453.

51 Carlyle, p. 105.

52 Kate Flint.. The Woman Reader 1837-1914. Oxford: Clarendon Press, 2002, pp. 191-209.

53 Flint, p. 200.

54 Fint, p. 202. 
Las comunidades británicas descritas en este trabajo, "outsiders" en la otredad de un país extranjero, buscaban maneras de afirmar su identidad nacional para mantener su cohesión como grupo y preservar los vínculos con la metrópoli. La presencia de estos volúmenes de y sobre William Shakespeare dan fe de la importante recepción que tuvo el clásico inglés en estos enclaves del sur de la Península Ibérica a finales del XIX. Si tradicionalmente se ha estudiado la recepción de la figura de Shakespeare a través de análisis críticos detallados de diferentes aspectos, como la lectura de sus sonetos o la asistencia a sus obras de teatro, cabe además, a nuestro juicio, ampliar este escenario valorando el rastro de su influencia durante el siglo XIX en toda la extensión del Imperio británico e incluso en enclaves ajenos a él en los que se instalaron importantes comunidades de habla intlesa. Tiffany Stern, en su capítulo "Shakespeare in Drama” afirma que "What affected eighteenth-century dramatists was not the actual Shakespeare but the works and the person that they were able to make him be" ${ }^{5}$, una afimación que podría extenderse al caso de las bibliotecas de la Faja Pirítica Ibérica en el siglo XIX, en la medida en que al intentar afirmar su propia identidad, le dieron a su vez a Shakespeare una nueva.

En su reseña al libro de Gail Marshall, Pollack-Pelzner afirma que a lo largo del siglo XIX Shakespeare fue canonizado, novelado, domesticado, afrancesado, germanizado o transformado en contemporáneo ${ }^{56}$. A esta lista podemos añadir que Shakespeare fue exportado a microcosmos coloniales en el extranjero, llegando a enclaves de explotación minera como Minas de Riotinto en la provincia de Huelva y a Mina de Santo Domingo en el Alentejo en Portugal y que fue catalogado en un sistema que repetía el de las bibliotecas victorianas existentes en Londres. La ocupación británica de tales pseudo-colonias se perdería con el tiempo, pero Shakespeare continuó como muestra de un legado imborrable.

55 Fiona Ritchie \& Peter Sabor (eds.). Shakespeare in the Eighteenth Century. Cambridge: Cambridge University Press, 2012, p. 142.

56 Daniel Pollarck-Pelzner, "Review on Shakespeare in the Nineteenth Century ed. by Gail Marshall. Victorian Studies, vol. 57, n.2, Winter (2015), 319-321. 
